\title{
Accelerated partial breast irradiation using multicatheter brachytherapy for select early-stage breast cancer: local control and toxicity
}

\author{
Seung-Gu Yeo*1,2, Juree Kim²,6, Geum-Hee Kwak33, Ji-Young Kim4, Kyeongmee Park5, Eun Seok Kim¹ and Sehwan Han³
}

\begin{abstract}
Background: To investigate the efficacy and safety of accelerated partial breast irradiation (APBI) via high-dose-rate (HDR) multicatheter interstitial brachytherapy for early-stage breast cancer.

Methods: Between 2002 and 2006, 48 prospectively selected patients with early-stage breast cancer received APBI using multicatheter brachytherapy following breast-conserving surgery. Their median age was 52 years (range 36-78). A median of $34 \mathrm{~Gy}$ (range 30-34) in 10 fractions given twice daily within 5 days was delivered to the tumor bed plus a 1$2 \mathrm{~cm}$ margin. Most (92\%) patients received adjuvant systemic treatments. The median follow-up was 53 months (range 36-95). Actuarial local control rate was estimated from surgery using Kaplan-Meier method.

Results: Local recurrence occurred in two patients. Both were true recurrence/marginal miss and developed in patients with close $(<0.2 \mathrm{~cm})$ surgical margin after 33 and 40 months. The 5 -year actuarial local recurrence rate was 4.6\%. No regional or distant relapse and death has occurred to date. Late Grade 1 or 2 late skin and subcutaneous toxicity was seen in 11 (22.9\%) and 26 (54.2\%) patients, respectively. The volumes receiving 100\% and 150\% of the prescribed dose were significantly higher in the patients with late subcutaneous toxicity $(p=0.018$ and 0.034 , respectively). Cosmesis was excellent to good in $89.6 \%$.
\end{abstract}

Conclusions: APBI using HDR multicatheter brachytherapy yielded local control, toxicity, and cosmesis comparable to those of conventional whole breast irradiation for select early-stage breast cancer. Patients with close resection margins may be ineligible for APBI.

\section{Background}

Over the last decades, breast-conserving surgery (BCS) followed by whole breast irradiation (WBI) became the standard of care for the treatment of early-stage breast cancer. However, the 5-6 weeks of conventional WBI are problematic for elderly patients, working women, and those who live a great distance from a radiotherapy facility [1]. In addition, controversies and logistical problems exist that are associated with integrating this prolonged course of WBI and systemic chemotherapy [2]. These make a barrier to the acceptance of breast conservation by patients or their physicians, and some patients do not receive WBI after BCS [3].

* Correspondence: md6630@daum.net

1 Department of Radiation Oncology, Soonchunhyang University College of Medicine, Cheonan, Korea

Full list of author information is available at the end of the article
The rationale underlying the accelerated partial breast irradiation (APBI) is that in-breast failure for select lowrisk patients occurs mostly in the immediate area of the tumor bed [4]. The risk of failing at a location remote from the tumor bed is very low; it occurs despite WBI and its rate is similar to that of new contralateral breast cancer $[5,6]$. Accordingly, standard elective irradiation of the entire breast for presumed occult disease can be replaced with partial breast irradiation. The reduction in irradiation volume allows the administration of a larger fraction dose in a shorter period without significant additional toxicity. In addition, WBI-induced cardiovascular mortality, which counteracted an increase in overall survival by adding WBI after BCS, may be reduced using APBI by avoiding radiation exposure to coronary vessels [7].

(C) 2010 Yeo et al; licensee BioMed Central Ltd. This is an Open Access article distributed under the terms of the Creative Commons AtB. Wed Central tribution License (http://creativecommons.org/licenses/by/2.0), which permits unrestricted use, distribution, and reproduction in any
medium, provided the original work is properly cited. 
Several centers have evaluated the feasibility and efficacy of APBI and produced evidences supporting the use of APBI for select early-stage breast cancer patients [810]. We pioneered APBI in our country, and this is the first report of long-term outcomes. The study investigated the efficacy and safety of APBI using high-dose-rate (HDR) multicatheter interstitial brachytherapy for select early-stage breast cancer patients and the endpoints were the local control rate, treatment toxicity, and cosmesis.

\section{Methods}

\section{Patients}

This study included 48 prospectively selected women with breast cancer in whom adjuvant radiotherapy was performed using interstitial brachytherapy alone after BCS. They were treated between May 2002 and December 2006, at the Inje University Sanggye Paik Hospital. We recommended interstitial brachytherapy for patients who met all of the following criteria: (1) age $>35$ years, (2) tumor size $\leq 4 \mathrm{~cm}$, (3) negative surgical margin, (4) negative axillary lymph nodes or singular positive node without extracapsular extension, (5) suitable breast anatomy for implantation, and (6) full recognition of possible increased risk of local failure. Patients with invasive lobular histology, extensive intraductal carcinoma, or multifocality were excluded. The study was approved by our institutional review board and written informed consent was obtained from the patients.

The median patient age was 52 years (range 36-78). Histological subtypes were invasive ductal carcinoma in $36(75.0 \%)$ patients, medullary carcinoma in $6(12.5 \%)$, ductal carcinoma in situ in 5 (10.4\%), and tubular carcinoma in $1(2.1 \%)$. The pathological T classification was Tis in 5 (10.4\%), T1 in 28 (58.3\%), and T2 in $15(31.3 \%)$. The pathological N classification was N0 in 44 (91.7\%) and N1 in 4 (8.3\%); one positive node without extracapsular extension was found out of 6-16 nodes retrieved. The pathological resection margin was clear $(\geq 0.2 \mathrm{~cm})$ in 42 $(87.5 \%)$ and close $(>0,<0.2 \mathrm{~cm})$ in $6(12.5 \%)$ patients. Further information on the patient, tumor, and treatment characteristics is given in Table 1.

\section{Treatments}

All patients underwent BCS with gross total resection of the primary tumor and a sentinel node biopsy $(\mathrm{n}=29$, $60.4 \%$ ) or level I/II axillary dissection $(\mathrm{n}=19,39.6 \%)$. Four titanium clips were positioned at the excision cavity boundaries: superiorly, inferiorly, medially and laterally. Four to six (median 5) guide needles were inserted during surgery. A single $(n=17,35.4 \%)$ or double $(n=31,64.6 \%)$ plane implant was performed. The needles were separated from each other by $1-1.5 \mathrm{~cm}$. The distance from the implant plane to the thoracic wall or overlying skin
Table 1: Patient, tumor, and treatment characteristics

\begin{tabular}{|c|c|c|}
\hline & & No (\%) \\
\hline \multicolumn{3}{|c|}{ Age } \\
\hline & Range & $36-78$ \\
\hline & Median & 52 \\
\hline \multicolumn{3}{|c|}{ Tumor size $(\mathrm{cm})$} \\
\hline & Range & $0.4-4.0$ \\
\hline & Median & 1.5 \\
\hline \multicolumn{3}{|c|}{ T classification } \\
\hline & Tis & $5(10.4)$ \\
\hline & $\mathrm{T} 1$ & $28(58.3)$ \\
\hline & $\mathrm{T} 2$ & $15(31.3)$ \\
\hline \multicolumn{3}{|c|}{$\mathrm{N}$ classification } \\
\hline & No & $44(91.7)$ \\
\hline & $\mathrm{N} 1$ & $4(8.3)$ \\
\hline \multicolumn{3}{|c|}{ Histological subtype } \\
\hline & Invasive ductal & $36(75.0)$ \\
\hline & Invasive medullary & $6(12.5)$ \\
\hline & Ductal carcinoma in situ & $5(10.4)$ \\
\hline & Invasive tubular & $1(2.1)$ \\
\hline \multicolumn{3}{|c|}{ Hormone receptor } \\
\hline & $\mathrm{ER}+$ and $\mathrm{PR}+$ & $34(70.8)$ \\
\hline & $\mathrm{ER}+$ and $\mathrm{PR}-$ & $3(6.3)$ \\
\hline & ER - and PR - & $11(22.9)$ \\
\hline \multicolumn{3}{|c|}{ Resection margin } \\
\hline & Clear $(\geq 0.2 \mathrm{~cm})$ & $42(87.5)$ \\
\hline & Close $(>0,<0.2 \mathrm{~cm})$ & $6(12.5)$ \\
\hline \multicolumn{3}{|c|}{ Radiation therapy } \\
\hline & 34 Gy (3.4 Gy/fraction) & $40(83.3)$ \\
\hline & 30 Gy (3.0 Gy/fraction) & $8(16.7)$ \\
\hline \multicolumn{3}{|c|}{ Systemic therapy } \\
\hline & $\begin{array}{l}\text { Chemotherapy + Hormonal } \\
\text { therapy }\end{array}$ & $24(50.0)$ \\
\hline & Hormonal therapy & $15(31.3)$ \\
\hline & Chemotherapy & $5(10.4)$ \\
\hline & None & $4(8.3)$ \\
\hline
\end{tabular}

Abbreviation: $\mathrm{ER}=$ estrogen receptor; $\mathrm{PR}=$ progesterone receptor.

should not be $<1 \mathrm{~cm}$. The needles were replaced with flexible catheters and fixed with buttons.

Radiotherapy was started after receiving complete histological reports, at an interval of 6-9 days after surgery. The radiotherapy was planned using the PLATO brachytherapy planning system (Nucletron BV, Veenendaal, The Netherlands). Two post-implant isocentric radiographs were taken on a simulator with variable 
angles and used for digitizing and three-dimensional reconstruction of the catheters and clips. The dose points were related to the active source positions, and they were placed at a given distance $(0.5-1 \mathrm{~cm})$ from the catheters. The distance and active source positions were defined individually for each catheter, considering the location of the clips. The size of the planning target volume was estimated in such a way that the reference dose points were $1-2 \mathrm{~cm}$ from the clips in each direction. Then, the dose points and geometry were optimized [11]. The median prescribed reference dose was $34 \mathrm{~Gy}(\mathrm{n}=40)$ in 10 fractions bid separated by a minimum 6-h interval within 5 days. Eight patients received 30 Gy in 10 fractions bid. Patients were treated in the supine position using the microSelectron HDR remote afterloading equipment with iridium-192 (Nucletron BV). Before each radiotherapy session, a radiation oncologist monitored the patients for complications and checked the catheter placement. If not all of the pathological criteria for sole interstitial brachytherapy were met, then the interstitial brachytherapy was converted to boost irradiation followed by a course of WBI and these patients were excluded from this study.

To estimate the skin dose, a flexible wire cross was positioned on the skin surface as representatively as possible above the active source positions. During the process of digitizing the implants, the dose points were also assessed with the help of two isocentric radiographs. Representative skin point doses were calculated and the maximum skin dose was documented for each patient.

Chemotherapy was given to $29(60.4 \%)$ patients starting within 9 days of the start of brachytherapy: cyclophosphamide, methotrexate, and 5-fluorouracil in 26 and doxorubicin, cyclophosphamide, and docetaxel in 3 . Thirtynine (81.3\%) patients received hormonal therapy: tamoxifen in 22 and an aromatase inhibitor in 17.

The patients were seen every 3 months for the first 2 years and every 6 months thereafter, with a physical examination, chest X-ray, and blood tests. Mammography and ultrasound examinations of the breast and abdomen were performed at 6 months after APBI and then yearly thereafter.

\section{Analysis}

To quantify the dose distributions, volume parameters and the dose homogeneity index (DHI) were calculated using dose-volume histograms. The volume parameters included the volumes receiving $100 \%$ and $150 \%$ of the prescribed dose $\left(\mathrm{V}_{100}\right.$ and $\mathrm{V}_{150}$, respectively). The DHI was calculated as $\left(\mathrm{V}_{100}-\mathrm{V}_{150}\right) / \mathrm{V}_{100}$, and was used to assess the dosimetric quality.

Local recurrence was defined as the recurrence of cancer in the treated breast proven histologically. A true recurrence/marginal miss was defined as a recurrence within or immediately adjacent to the primary tumor site. An elsewhere recurrence was defined as a local recurrence detected at least $2 \mathrm{~cm}$ from the surgical clips [12]. The actuarial rate of local recurrence was estimated from the date of surgery using the Kaplan-Meier method.

The cosmetic evaluation was based on the standards set forth in the Harvard criteria, which consisted of a fourtiered grading system: excellent, good, fair, and poor [13]. Late toxicity of the skin and subcutaneous tissue was scored according to the Radiation Therapy Oncology Group (RTOG)/European Organization for Research and Treatment of Cancer late radiation morbidity scoring scheme [14]. The cosmesis and toxicity scores recorded at the last follow-up were analyzed. To analyze the association between the dosimetric parameters or chemotherapy use and treatment toxicity, $t$-test, Fisher's exact test, or the chi-square test was used, as appropriate. A p-value of $<0.05$ was deemed statistically significant. All statistical tests were performed using SPSS software (release 14.0; SPSS Inc., Chicago, IL, USA).

\section{Results \\ Local control}

The median follow-up period was 53 months (range 3695 ) and there was no death. Local recurrence occurred in two patients 33 and 40 months after surgery. Both were true recurrence/marginal miss and developed in patients with close surgical margin (Table 2). The 5-year actuarial local recurrence rate was $4.6 \%$. No regional nodal or distant metastasis was detected. The patients with recurrences received salvage surgery and all patients were alive without evidence of disease at the last follow-up.

\section{Dosimetry, toxicity, and cosmesis}

The mean $\mathrm{V}_{100}$ and $\mathrm{V}_{150}$ values were $44.7 \pm 17.9 \mathrm{~cm}^{3}$ (range 12-101) and $22.8 \pm 8.3 \mathrm{~cm}^{3}$ (range 5-46), respectively. The mean DHI was $0.5 \pm 0.03$ (range 0.44-0.57). The maximum skin dose ranged from 12-69\% (median 39\%) of the prescribed dose.

Early side effects were usually mild and the breast pain, edema, or erythema subsided with conservative management. Grade 1 and 2 late skin toxicity occurred in 8 (16.7\%) and $3(6.3 \%)$ patients, respectively. Grade 1 and 2 late subcutaneous toxicity developed in 19 (39.6\%) and 7 (14.6\%) patients, respectively. Asymptomatic fat necrosis was detected on routine follow-up mammography in 5 (10.4\%) patients, but required no surgical intervention. Dosimetric parameters like the $\mathrm{V}_{100}, \mathrm{~V}_{150}$, and DHI did not differ significantly according to the occurrence of late skin toxicity. $\mathrm{V}_{100}$ and $\mathrm{V}_{150}$ were significantly higher in the patients with late subcutaneous toxicity $(\mathrm{p}=0.018$ and 0.034 , respectively) (Table 3 ). The maximum skin dose 
Table 2: Characteristic of the patients with local recurrence

\begin{tabular}{ccccccccccccc}
\hline No & Age & Pathology & $\begin{array}{c}\text { Tumor } \\
\text { size }(\mathbf{c m})\end{array}$ & pN & ER & PR & RM & $\begin{array}{c}\text { Radiation } \\
\text { therapy }\end{array}$ & $\begin{array}{c}\text { Systemic } \\
\text { therapy }\end{array}$ & $\begin{array}{c}\text { Failure } \\
\text { failure (mo) }\end{array}$ \\
\hline 1 & 52 & DCIS & 1.8 & 0 & + & - & Close & 34 Gy & TAM & TR/MM \\
surgery \\
after salvage \\
surgery (mo)
\end{tabular}

Abbreviation: $\mathrm{pN}=$ pathological nodal classification; $\mathrm{ER}=$ estrogen receptor; $\mathrm{PR}=$ progesterone receptor; $\mathrm{RM}=$ resection margin; $\mathrm{DCIS}=$ ductal carcinoma in situ; $\mathrm{IDC}=$ invasive ductal carcinoma; TAM = tamoxifen; $C M F=$ cyclophosphamide, methotrexate, 5 -fluorouracil; TR/MM = true recurrence/marginal miss; $B C S=$ breast-conserving surgery; $M R M=$ modified radical mastectomy. 
Table 3: Comparison of dosimetric parameters according to the late treatment toxicity

\begin{tabular}{|c|c|c|c|c|c|c|c|c|}
\hline Toxicity & & No (\%) & $V_{100}\left(\mathrm{~cm}^{3}\right)$ & $P \neq$ & $V_{150}\left(\mathrm{~cm}^{3}\right)$ & $P \neq$ & DHI & $P \neq$ \\
\hline \multicolumn{9}{|l|}{ Skin } \\
\hline & Yes* & $11(22.9)$ & $51.3 \pm 12.1$ & 0.221 & $25.7 \pm 6.2$ & 0.222 & $0.51 \pm 0.03$ & 0.105 \\
\hline & No & $37(77.1)$ & $43.0 \pm 18.9$ & & $22.0 \pm 8.7$ & & $0.49 \pm 0.03$ & \\
\hline \multicolumn{9}{|c|}{ Subcutaneous tissue } \\
\hline & Yes $^{\dagger}$ & $26(54.2)$ & $50.2 \pm 18.2$ & 0.018 & $25.1 \pm 8.1$ & 0.034 & $0.50 \pm 0.03$ & 0.099 \\
\hline & No & $22(45.8)$ & $37.5 \pm 15.0$ & & $19.9 \pm 7.8$ & & $0.48 \pm 0.03$ & \\
\hline \multicolumn{9}{|c|}{ Fat necrosis } \\
\hline & Yes & $5(10.4)$ & $40.4 \pm 18.7$ & 0.529 & $21.0 \pm 9.8$ & 0.560 & $0.48 \pm 0.02$ & 0.465 \\
\hline & No & $43(89.6)$ & $45.4 \pm 17.9$ & & $23.1 \pm 8.1$ & & $0.49 \pm 0.03$ & \\
\hline
\end{tabular}

was $43 \pm 12 \%$ and $37 \pm 11 \%$ in the patients with and without late skin toxicity, respectively $(\mathrm{p}=0.190)$. The rates of late treatment toxicities did not differ according to the use of chemotherapy. Cosmesis was excellent $(n=34)$ or good $(n=9)$ in $89.6 \%$ of the patients. No one had poor cosmesis.

\section{Discussion}

Long-term results of the phase II multicenter APBI trials for select early-stage breast cancer were recently reported [8-10,15]. In the RTOG phase II trial [9], 66 patients received HDR brachytherapy (34 Gy in 3.4 Gy bid for 5 days) and 33 patients received low-dose-rate brachytherapy (45 Gy). The estimated 5-year local recurrence rate was $4 \%$ (3\% in HDR and $6 \%$ in low-dose-rate) after a median follow-up of 7 years. In the German-Austrian phase II trial [10], 175 patients received pulsed-dose-rate brachytherapy (49.8 Gy) and 99 received HDR brachytherapy (32 Gy in 4 Gy bid for 4 days). After a median follow-up of 32 months, the 3-year local recurrence rate was $0.4 \%$. In the single-institution phase II trial conducted in Hungary [8], 45 patients received HDR brachytherapy, either 36.4 Gy $(\mathrm{n}=37)$ or $30.3 \mathrm{~Gy}(\mathrm{n}=8)$ in seven fractions for 4 days. After a median follow-up of 81 months, the 5-year local recurrence rate was $4.4 \%$, which was not significantly different from that for WBI in a retrospective comparative analysis. Overall, recently published APBI studies using multicatheter interstitial brachytherapy reported annual local recurrence rates below $1 \%$, which is equivalent to the outcomes of WBI $[8,16]$. We found a $4.6 \% 5$-year local recurrence rate, which translates to an annual recurrence rate of $0.9 \%$, and is not different from those of other institutions.
Patients who have a substantial chance of harboring residual disease located a significant distance from the edge of the excision cavity or who potentially have multicentric disease have been precluded from APBI trials. The eligibility criteria of the RTOG trial included unicentricity, T1 or T2 $\leq 3 \mathrm{~cm})$, infiltrating nonlobular carcinoma, pathologically negative margin, N0 or N1 without extracapsular extension, and no extensive intraductal component [9]. The eligibility criteria of the GermanAustrian trial included tumor diameter $\leq 3 \mathrm{~cm}$, clear resection margin $(\geq 0.2 \mathrm{~cm})$, N0 or singular nodal micrometastasis, estrogen and/or progesterone receptor positive, and $\geq 35$ years [10]; patients were excluded if multifocality, poor differentiation, an extensive intraductal component, or lymphovascular invasion existed. Regarding resection margin, the American Brachytherapy Society recommended a negative margin, whereas the American Society of Breast Surgeons recommended a margin of at least $0.2 \mathrm{~cm}$ [4]. We experienced two local recurrences which were true recurrence/marginal miss and occurred to the patients with close resection margin. Positive margin status is generally accepted as a major risk factor for local recurrence after BCS and radiotherapy, and the width of clear surgical margins significantly influences local tumor control [17]. At least $0.2 \mathrm{~cm}$ tumor-free margins are deemed acceptable in some APBI trials $[10,18]$, but others also successfully treated patients with close margins by APBI $[8,9]$. However, recently published recommendations for APBI selection criteria categorized patients with close margins as an intermediaterisk group, as there are only limited data supporting the use of APBI for these patients [19]. Our results may indicate that close margins should be an exclusion criterion for APBI trials. 
The most commonly prescribed dose of sole HDR brachytherapy for breast cancer is 34 Gy in ten fractions bid, which is equivalent to a 46 Gy tumor dose using the standard WBI scheme (2 Gy per day, 5 days per week) [20]. Whether the higher local recurrence risk after incomplete tumor excision can be counterbalanced by an additional boost radiotherapy following WBI has not been demonstrated clearly [21]; however, a HDR brachytherapy boost of 13.2 Gy in three fractions following $50 \mathrm{~Gy} / 25$ fractions WBI produced favorable local control for close to positive margins [22]. The traditional two X-ray film localization technique used in both this study and other recent reports [8-10] cannot define the actual extent of the target volume and it relates the prescribed dose to the geometry of the implant and not to the target volume. To localize the irregular three-dimensional shape of the target volume and the normal tissue structures correctly and to adapt the reference isodose surface to the shape of this target volume, the utility of brachytherapy planning based on computed tomography imaging has been investigated [23,24]. With sophisticated computed tomography-based implantation and threedimensional planning system, the target volume definition considering inadequate resection margin foci and the modulation of a higher dose to cover this region might be efficient for those patients who have an insufficient resection margin.

Compared to the postoperative implantation [8-10], intraoperative implantation has the advantages of direct visualization of the excision cavity and shorter local treatment period including surgery and radiotherapy [15]. One disadvantage is the inability to select patients properly for implantation based on definitive pathological findings; however, brachytherapy was used as boost radiotherapy before WBI when the pathology indicated that the patient was unsuitable for brachytherapy alone.

Preliminary guidelines designed to reduce the toxicity of HDR interstitial brachytherapy have been reported [25]. First, ideally, less than $60 \%$ of the normal whole breast volume should receive $\geq 50 \%$ of the prescribed dose. In this respect, Asian women are at a disadvantage due to their relatively small breasts compared to European and American women, and this might be one of the reasons why APBI has not been actively investigated for them [26]. To satisfy this recommendation, the implementation of computed tomography-based three-dimensional planning would be advantageous. Second, one must minimize hot spots $\left(\mathrm{V}_{150}\right)$ and maintain DHI $>0.75$. We found that a higher $\mathrm{V}_{100}$ or $\mathrm{V}_{150}$ was associated with a significantly higher rate of late subcutaneous toxicity. Mean DHI was low as 0.5 , however dose inhomogeneity can make a positive contribution in terms of the tumor control probability [27]. Third, the dose delivered to the skin and chest wall should be less than the prescribed dose. We selected patients with sufficient breast tissue anterior to the tumor and the maximum skin dose was restricted to below $70 \%$ of the prescribed dose. Finally, one must proceed with caution if chemotherapy is to be given following APBI. Adriamycin-based chemotherapy after APBI was reportedly related to worse toxicity and cosmesis [25]. We started chemotherapy (mostly not adriamycin-based) during or right after APBI and the chemotherapy use did not affect late toxicity. Previously, we reported the non-inferior safety of concurrent chemoradiotherapy compared to sequential chemoradiotherapy using WBI for early-stage breast cancer [2]. However, the use of larger fraction dose in APBI compared to WBI may necessitate careful administration of chemotherapy. Furthermore, normal tissue changes after APBI have been documented to evolve over time; some endpoint measures (cosmesis, edema, erythema, and breast pain) improved with time, while others (fat necrosis, subcutaneous fibrosis, and telangiectasia) worsened $[28,29]$. These findings underscore the extended period needed to monitor APBI-related late treatment toxicity, and some patients in this study may need more follow-up to fully evaluate late toxicity.

A few details of the methods need to be mentioned. First, the relatively small breasts in the patients caused concern for treatment toxicity owing to the high irradiated volume/ipsilateral breast volume ratio. Thus, a schedule of 30 Gy in 10 fractions was tried for the first eight patients. After the feasibility and safety of APBI was verified for these eight patients, a dose of $34 \mathrm{~Gy}$ in 10 fractions was adopted for the remaining patients. Second, the number of catheters used was small (median 5), with a single plane implant in $17(35.4 \%)$ patients. We tried to remove a tumor and at least $1 \mathrm{~cm}$ margin, while at the same time trying to minimize the total excision volume for cosmesis. APBI started shortly (6-9 days) after surgery, and the hemovac drainage was maintained until APBI completed. Accordingly, the excision cavity was less likely to accumulate a hematoma or seroma, and the mean $\mathrm{V}_{100}$ was relatively small, at $44.7 \mathrm{~cm}^{3}$. A single plane implant was used when the excision cavity was small and flat. However, an increase in catheter number, less use of a single plane implant or computed tomography-based three-dimensional dose planning would be necessary to enhance dose homogeneity.

\section{Conclusions}

In conclusion, APBI using HDR multicatheter interstitial brachytherapy for early-stage breast cancer yielded local control, toxicity, and cosmesis comparable to those of other recent APBI trials or conventional WBI. Our results support the suggestion that APBI is a viable option for select patients with breast cancer. Patients with close resection margins may be ineligible for APBI, and studies 
of novel brachytherapy techniques should be pursued to optimize APBI outcomes.

\section{List of abbreviations}

BCS: breast-conserving surgery; WBI: whole breast irradiation; APBI: accelerated partial breast irradiation; HDR: high-dose-rate; DHI: dose homogeneity index; $\mathrm{V}_{100}$ and $\mathrm{V}_{150}$ : volumes receiving $100 \%$ and $150 \%$ of the prescribed dose, respectively; RTOG: radiation therapy oncology group.

\section{Competing interests}

The authors declare that they have no competing interests.

\section{Authors' contributions}

SGY, SH, JK: conception and design, acquisition, analysis and interpretation of data; GHK, JYK, KP: acquisition, analysis and interpretation of data; ESK: analysis and interpretation of data. All the listed authors have been involved in drafting or in revising the manuscript. All authors read and approved the final manuscript.

\section{Author Details}

'Department of Radiation Oncology, Soonchunhyang University College of Medicine, Cheonan, Korea, ${ }^{2}$ Department of Radiation Oncology, Inje University Sanggye Paik Hospital, Seoul, Korea, ${ }^{3}$ Department of Surgery, Inje University Sanggye Paik Hospital, Seoul, Korea, ${ }^{4}$ Department of Radiology, Inje University Sanggye Paik Hospital, Seoul, Korea, ${ }^{5}$ Department of Pathology, Inje University Sanggye Paik Hospital, Seoul, Korea and ${ }^{6}$ Department of Radiation Oncology, Kwandong University Jeil Hospital, Seoul, Korea

Received: 9 April 2010 Accepted: 19 June 2010

Published: 19 June 2010

\section{References}

1. Hebert-Croteau N, Brisson J, Latreille J, Blanchette C, Deschenes L: Compliance with consensus recommendations for the treatment of early stage breast carcinoma in elderly women. Cancer 1999, 85:1104-1113

2. Han S, Kim J, Sohn S, Kwak GH, Kim JY, Park K: Feasibility of concurrent adjuvant chemotherapy and radiotherapy after breast-conserving surgery in early breast cancer. J Surg Oncol 2007, 95:45-50.

3. Morrow M, White J, Moughan J, Owen J, Pajack T, Sylvester J, Wilson JF, Winchester $\mathrm{D}$ : Factors predicting the use of breast-conserving therapy in stage I and II breast carcinoma. J Clin Oncol 2001, 19:2254-2262.

4. Arthur DW, Vicini FA: Accelerated partial breast irradiation as a part of breast conservation therapy. J Clin Oncol 2005, 23:1726-1735.

5. Veronesi U, Cascinelli N, Mariani L, Greco M, Saccozzi R, Luini A, Aguilar M, Marubini E: Twenty-year follow-up of a randomized study comparing breast-conserving surgery with radical mastectomy for early breast cancer. N Engl J Med 2002, 347:1227-1232.

6. Morrow M: Rational local therapy for breast cancer. N Eng/ J Med 2002, 347:1270-1271

7. Clarke M, Collins R, Darby S, Davies C, Elphinstone P, Evans E, Godwin J, Gray R, Hicks C, James S, MacKinnon E, McGale P, McHugh T, Peto R, Taylor C, Wang Y: Effects of radiotherapy and of differences in the extent of surgery for early breast cancer on local recurrence and 15-year survival: an overview of the randomised trials. Lancet 2005, 366:2087-2106.

8. Polgar C, Major T, Fodor J, Nemeth G, Orosz Z, Sulyok Z, Udvarhelyi N, Somogyi A, Takacsi-Nagy Z, Lovey K, Agoston P, Kasler M: High-dose-rate brachytherapy alone versus whole breast radiotherapy with or without tumor bed boost after breast-conserving surgery: seven-year results of a comparative study. Int J Radiat Oncol Biol Phys 2004, 60:1173-1181.

9. Arthur DW, Winter K, Kuske RR, Bolton J, Rabinovitch R, White J, Hanson WF, Wilenzick RM, McCormick B: A Phase II trial of brachytherapy alone after lumpectomy for select breast cancer: tumor control and survival outcomes of RTOG 95-17. Int J Radiat Oncol Biol Phys 2008, 72:467-473.
10. Ott OJ, Hildebrandt G, Potter R, Hammer J, Lotter M, Resch A, Sauer R, Strnad V: Accelerated partial breast irradiation with multi-catheter brachytherapy: Local control, side effects and cosmetic outcome for 274 patients. Results of the German-Austrian multi-centre trial. Radiother Oncol 2007, 82:281-286.

11. der LV, Prins TPE: Introduction to HDR brachytherapy optimisation. In Brachytherapy from radium to optimization Edited by: Mould RF. Nucletron International; 1994:331-351.

12. Recht A, Silver B, Schnitt S, Connolly J, Hellman S, Harris JR: Breast relapse following primary radiation therapy for early breast cancer. I. Classification, frequency and salvage. Int J Radiat Oncol Biol Phys 1985, 11:1271-1276

13. Harris JR, Levene MB, Svensson G, Hellman S: Analysis of cosmetic results following primary radiation therapy for stages I and II carcinoma of the breast. Int J Radiat Oncol Biol Phys 1979, 5:257-261.

14. Cox JD, Stetz J, Pajak TF: Toxicity criteria of the Radiation Therapy Oncology Group (RTOG) and the European Organization for Research and Treatment of Cancer (EORTC). Int J Radiat Oncol Biol Phys 1995, 31:1341-1346

15. Aristei C, Palumbo I, Cucciarelli F, Cavalli A, Tarducci R, Raymondi C, Perrucci E, Cavaliere A, Latini P, Rulli A: Partial breast irradiation with interstitial high-dose-rate brachytherapy in early breast cancer: results of a phase II prospective study. Eur J Surg Oncol 2009, 35:144-150.

16. Polgar C, Major T: Current status and perspectives of brachytherapy for breast cancer. Int J Clin Oncol 2009, 14:7-24.

17. Schnitt SJ, Abner A, Gelman R, Connolly JL, Recht A, Duda RB, Eberlein TJ, Mayzel K, Silver B, Harris JR: The relationship between microscopic margins of resection and the risk of local recurrence in patients with breast cancer treated with breast-conserving surgery and radiation therapy. Cancer 1994, 74:1746-1751.

18. Polgar C, Fodor J, Major T, Nemeth G, Lovey K, Orosz Z, Sulyok Z, TakacsiNagy Z, Kasler M: Breast-conserving treatment with partial or whole breast irradiation for low-risk invasive breast carcinoma--5-year results of a randomized trial. Int J Radiat Oncol Biol Phys 2007, 69:694-702.

19. Polgar C, Van Limbergen E, Potter R, Kovacs G, Polo A, Lyczek J, Hildebrandt G, Niehoff P, Guinot JL, Guedea F, Johansson B, Ott OJ, Major $T$, Strnad V: Patient selection for accelerated partial-breast irradiation (APBI) after breast-conserving surgery: recommendations of the Groupe Europeen de Curietherapie-European Society for Therapeutic Radiology and Oncology (GEC-ESTRO) breast cancer working group based on clinical evidence (2009). Radiother Oncol 94:264-273.

20. Buchholz TA, Kuerer HM, Strom EA: Is partial breast irradiation a step forward or backward? Semin Radiat Oncol 2005, 15:69-75.

21. Jones HA, Antonini N, Hart AA, Peterse JL, Horiot JC, Collin F, Poortmans PM, Oei SB, Collette L, Struikmans H, Van den Bogaert WF, Fourquet A, Jager JJ, Schinagl DA, Warlam-Rodenhuis CC, Bartelink H: Impact of pathological characteristics on local relapse after breast-conserving therapy: a subgroup analysis of the EORTC boost versus no boost trial. J Clin Oncol 2009, 27:4939-4947.

22. Guinot JL, Roldan S, Maronas M, Tortajada I, Carrascosa M, Chust ML, Estornell M, Mengual $\mathrm{J}$, Arribas L: Breast-conservative surgery with close or positive margins: can the breast be preserved with high-doserate brachytherapy boost? Int J Radiat Oncol Biol Phys 2007, 68:1381-1387.

23. Major T, Frohlich G, Lovey K, Fodor J, Polgar C: Dosimetric experience with accelerated partial breast irradiation using image-guided interstitial brachytherapy. Radiother Oncol 2009, 90:48-55.

24. Aristei C, Tarducci R, Palumbo I, Cavalli A, Corazzi F, Rulli A, Raymondi C, Latini P: Computed tomography for excision cavity localization and 3Dtreatment planning in partial breast irradiation with high-dose-rate interstitial brachytherapy. Radiother Oncol 2009, 90:43-47.

25. Wazer DE, Kaufman S, Cuttino L, DiPetrillo T, Arthur DW: Accelerated partial breast irradiation: an analysis of variables associated with late toxicity and long-term cosmetic outcome after high-dose-rate interstitial brachytherapy. Int J Radiat Oncol Biol Phys 2006, 64:489-495

26. Mitsumori M, Hiraoka M: Current status of accelerated partial breast irradiation. Breast Cancer 2008, 15:101-107.

27. Powell SN: The radiobiology of accelerated partial breast irradiation. In Accelerated partial breast irradiation: techniques and clinical implementation 1st edition. Edited by: Wazer DE, Arthur DW, Vicini FA. Springer; 2006:60-61. 
28. Benitez PR, Chen PY, Vicini FA, Wallace M, Kestin L, Edmundson $G$, Gustafson G, Martinez A: Partial breast irradiation in breast conserving therapy by way of intersitial brachytherapy. Am J Surg 2004, 188:355-364.

29. Chen PY, Vicini FA, Benitez P, Kestin LL, Wallace M, Mitchell C, Pettinga J, Martinez AA: Long-term cosmetic results and toxicity after accelerated partial-breast irradiation: a method of radiation delivery by interstitial brachytherapy for the treatment of early-stage breast carcinoma. Cancer 2006, 106:991-999.

doi: 10.1186/1748-717X-5-56

Cite this article as: Yeo et al., Accelerated partial breast irradiation using multicatheter brachytherapy for select early-stage breast cancer: local control and toxicity Radiation Oncology 2010, 5:56

Submit your next manuscript to BioMed Centra and take full advantage of:

- Convenient online submission

- Thorough peer review

- No space constraints or color figure charges

- Immediate publication on acceptance

- Inclusion in PubMed, CAS, Scopus and Google Scholar

- Research which is freely available for redistribution

Submit your manuscript at www.biomedcentral.com/submit 